\begin{tabular}{|c|l|}
\hline Title & Electric-field-temperature phase diagram of an antiferroelectric liquid crystal \\
\hline Author(s) & Orihara, H.; Naruse, Y.; Y agyu, M.; Fajar, A.; Uto, S. \\
\hline Citation & $\begin{array}{l}\text { PHY SICAL REVIEW E, 72, 040701(R) } \\
\text { https://doi.org/L0.1103/PhysRevE.72.040701 }\end{array}$ \\
\hline Issue Date & 2005 \\
\hline Doc URL & http://hdl.handle.net/2115/5821 \\
\hline Rights & Copyright $\odot 2005$ A merican Physical Society \\
\hline Type & article \\
\hline File Information & PRE72-4.pdf \\
\hline
\end{tabular}

Instructions for use 


\title{
Electric-field-temperature phase diagram of an antiferroelectric liquid crystal
}

\author{
H. Orihara, ${ }^{1}$ Y. Naruse, ${ }^{2}$ M. Yagyu, ${ }^{2}$ A. Fajar, ${ }^{2}$ and S. Uto ${ }^{3}$ \\ ${ }^{1}$ Department of Applied Physics, Hokkaido University, Sapporo 060-8628, Japan \\ ${ }^{2}$ Department of Applied Physics, Nagoya University, Nagoya 464-8603, Japan \\ ${ }^{3}$ Department of Electrical Engineering, Osaka Institute of Technology, Osaka 535-8585, Japan
}

(Received 11 April 2005; published 28 October 2005)

\begin{abstract}
We present a precise electric-field-temperature phase diagram of an antiferroelectric liquid crystal with a short pitch $\mathrm{Sm}-C_{\alpha}^{*}$ phase. This was obtained by using a photoelastic modulator. A unique field-induced phase was found inside the Sm- $C_{\alpha}^{*}$ phase, which displayed low birefringence. Two tricritical points related to the phase were also observed. In addition, numerical calculations were made based on the discrete phenomenological model. The numerical results reproduced the experimental ones and it was clarified that the phase has a three-layer structure without spatial modulation.
\end{abstract}

DOI: 10.1103/PhysRevE.72.040701

PACS number(s): 64.70.Md, 61.30.Cz

Many phases have been found in antiferroelectric smectic liquid crystals. The variety can be ascribed to the interlayer interactions between the tilts of molecules. The $\mathrm{Sm}-C_{\alpha}^{*}$ phase with a short pitch helix arises from competition between nearest-neighbor (NN) and next-nearest-neighbor (NNN) interactions [1], and the distorted four-layer phase $\left(\mathrm{Sm}-C_{\mathrm{FI} 2}^{*}\right)$ and the distorted three-layer phase $\left(\mathrm{Sm}-C_{\mathrm{FI} 1}^{*}\right)$ from NN nonlinear interaction [2]. A phase diagram in a parameter space of coefficients in the free energy was obtained on the basis of a discrete phenomenological model. As the temperature changes, the interactions change so that sequential transitions take place. Consequently, by changing the temperature one can obtain information on the interactions. In addition, using an electric field can also be a good method for investigating the interactions because in chiral smectic liquid crystals the electric field couples with the order parameter representing the amplitude and phase of the molecular tilt in each layer. The electric-field effect has been extensively studied, mainly by using $D-E$ hysteresis loops, optical transmission measurements under ac electric fields, and conoscopic observations [3]. However, these experiments are not detailed enough to precisely detect small orientational changes in molecules over a wide range of temperatures and electric fields.

We adopted an optical system using a photoelastic modulator (PEM), by means of which we could simplify the simultaneous determination of the birefringence, $n_{\mathrm{a}}$, and the tilt angle of the optical axis, $\Theta$. Note that $\Theta$ used in this paper is different from the molecular tilt angle. By using the PEM system, we studied the electric-field-temperature $(E-T)$ phase diagram of 4-(1-methylheptyloxycarbonyl)-phenyl-4'-octylcarbonyloxybiphenyl-4-carboxylate (MHPOCBC), a compound exhibiting the phase sequence $\mathrm{Sm}-A\left(105.5^{\circ} \mathrm{C}\right) \mathrm{Sm}-C_{\alpha}^{*}\left(99.5^{\circ} \mathrm{C}\right) \mathrm{Sm}-C_{A}^{*}$. An $E-T$ phase diagram has already been made from optical transmittance measurements by Hiraoka et al. [4], and it was pointed out that there were some field-induced anomalies during the lowtemperature part of the Sm- $C_{\alpha}^{*}$ phase. Recently, however, the $\mathrm{Sm}-C_{\alpha}^{*}$ phase of MHPOCBC has been found to have a nanoscale incommensurate pitch [5]. The pitch decreased from $\approx 3$ to $\approx 2.6$ layers on cooling during the $\mathrm{Sm}-C_{\alpha}^{*}$ phase.
Here, we experimentally make a precise $E-T$ phase diagram and clarify the above field-induced anomalies in the $\mathrm{Sm}-C_{\alpha}^{*}$ phase on the basis of the discrete model.

The cells used in the experiments were of the usual sandwich type, consisting of two parallel glass substrates with inner surfaces coated with indium tin oxide (ITO). An alignment layer of polyimide was deposited on the ITO. In order to obtain better homogeneous alignment, the sample was submitted for $1 \mathrm{~h}$ to a low-frequency ac electric field around the $\operatorname{Sm} A-\operatorname{Sm} C_{\alpha}^{*}$ phase-transition point. The cell thickness was $22.6 \mu \mathrm{m}$, measured by an optical interference method. A He-Ne laser $(632.8 \mathrm{~nm})$ was used as a light source, and the beam propagation direction was perpendicular to the cell surfaces and parallel to the electric field (Fig. 1). In the measurements, the temperature was gradually decreased in steps and at each temperature the electric field was changed in steps. The total measuring points were 141 (temperatures) $\times 101$ (electric fields $)=14241$, and the total time was about $34 \mathrm{~h}$. The experimental procedure for the simultaneous measurements of the birefringence and the tilt of the optical axis will be described elsewhere.

Since it was difficult to obtain the absolute value of the birefringence in our experiment, we show the change from the value at the $\mathrm{Sm}-A$ to $\mathrm{Sm}-C_{\alpha}^{*}$ transition point without any electric field, $\Delta n_{\mathrm{a}}$, hereafter. The temperature and field dependence of the $\Delta n_{\mathrm{a}}$ in MHPOCBC is shown in Figs. 2(a) (surface plot) and 2(b) (corresponding contour plot), where $T_{\mathrm{c}}$ is the $\mathrm{Sm}-A$ to $\mathrm{Sm}-C_{\alpha}^{*}$ transition temperature. The phase

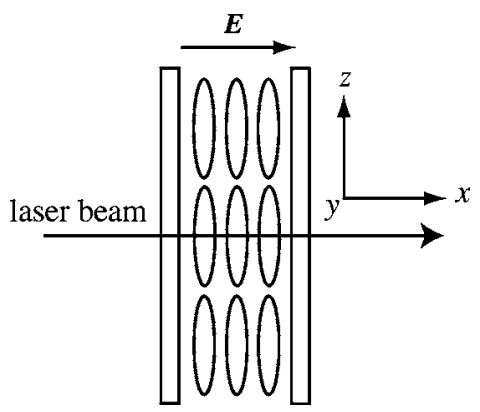

FIG. 1. Experimental geometry using a homogeneous cell. 


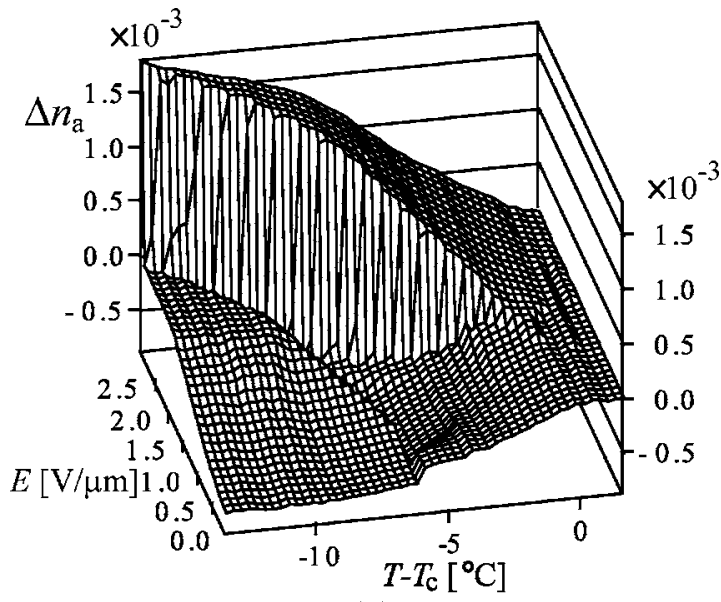

(a)

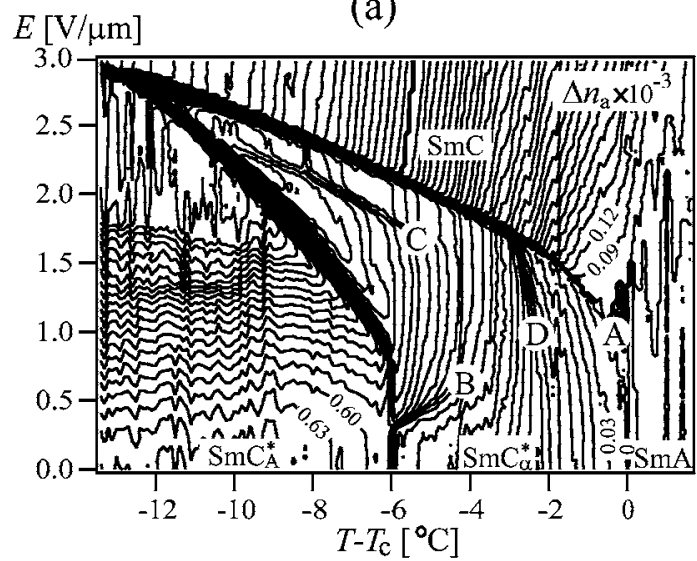

(b)

FIG. 2. $E-T$ phase diagram of the birefringence in MHPOCBC. (a) Surface plot and (b) contour plot, where the interval of lines is $0.03 \times 10^{-3}$

boundaries between $\mathrm{Sm}-A(\mathrm{Sm}-C)$ and $\mathrm{Sm}-C_{\alpha}^{*}$, and $\mathrm{Sm}-C_{\alpha}^{*}$ and $\mathrm{Sm}-C_{A}^{*}$, are clearly seen in Fig. 2(b). Note that contour lines become dense at the boundaries. In the $\mathrm{Sm}-A$ to $\mathrm{Sm}-C_{\alpha}^{*}$ transition there exists a tricritical point (designated as A), where the $\Delta n_{\mathrm{a}}$ begins to jump discontinuously. This tricritical point has already been found and investigated in detail by Bourny et al. [6]. There are three anomalies inside the $\mathrm{Sm}-C_{\alpha}^{*}$ phase (designated as B, C, and D). Anomaly B has already been reported by Hiraoka et al. [4], but not anomalies $\mathrm{C}$ and D. Furthermore, they reported that there were additional anomalies above the anomaly $\mathrm{B}$ and this was the evidence of a field-induced devil's staircase. However, in our measurements nothing was observed. As for the anomalies B and $\mathrm{C}$, it should be stressed that the birefringence decreases between them, as shown in Fig. 2(a), and the changes in the anomalies decrease with increasing temperature. Quite recently, Panov et al. made a precise birefringence measurement using a PEM in a homeotropically aligned cell of MHPOCBC and showed that the birefringence increases as the field is raised in the low-temperature region [8]. This result is compatible with ours in that the birefringence decreases because the measured planes are different; in the former, it is parallel to the smectic layers while in the latter it is perpendicular. The anomaly $\mathrm{D}$ starts from the phase

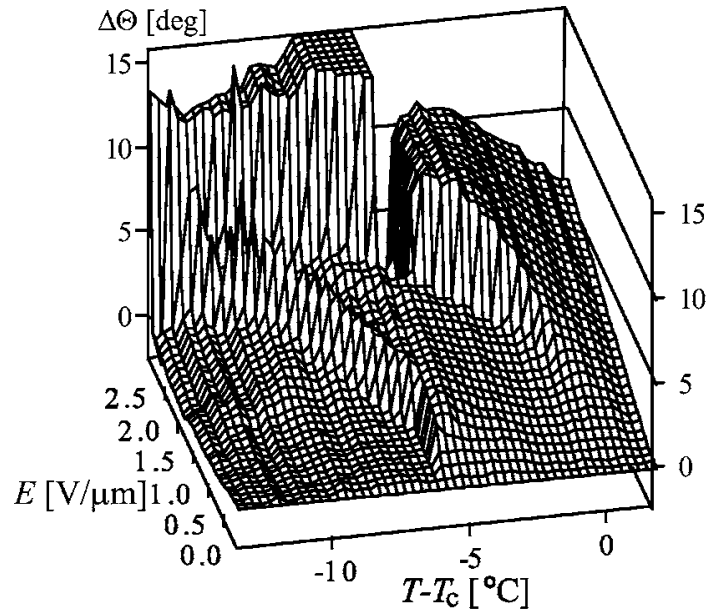

(a)

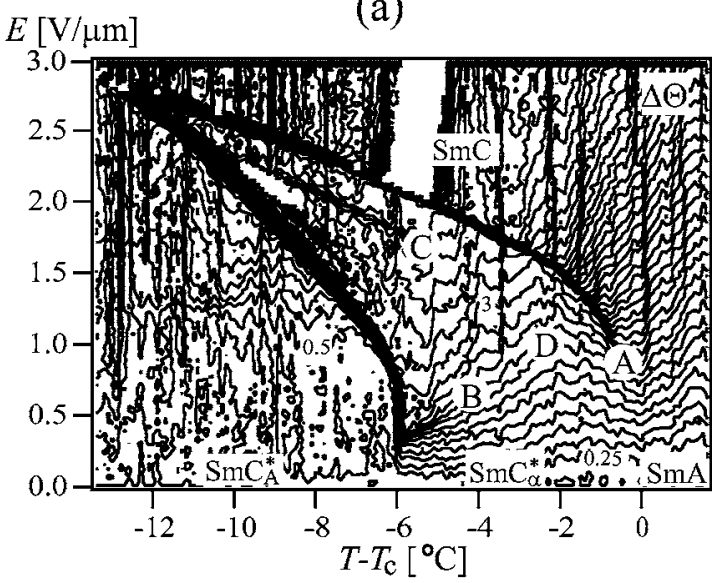

(b)

FIG. 3. $E$-T phase diagram of the optical tilt in MHPOCBC. (a) Surface plot and (b) contour plot, where the interval of lines is $0.25^{\circ}$.

boundary between $\mathrm{Sm}-A(\mathrm{Sm}-C)$ and $\mathrm{Sm}-C_{\alpha}^{*}$ and seems to disappear far from it.

The tilt angle of the optical axis $\theta$ could be obtained without uncertainty. However, we should note the fact that the smectic layers rotate after applying fields [7]. Also in our experiments, at each temperature $\Theta$ s before and after applying electric fields were slightly different ( at most $0.1^{\circ}$ ) so that $\Theta$ measured at $E=0$ was not independent of the temperature, though the optical axis should be always normal to the smectic layer in $\mathrm{Sm}-A, \mathrm{Sm}-C_{\alpha}^{*}$, and $\mathrm{Sm}-C_{A}^{*}$. Therefore, we used $\Delta \Theta \equiv \Theta(E)-\Theta(0)$ at each temperature. Figure 3 shows $\Delta \theta$, where we omitted some data in the plots because the retardation corresponding to them was close to 0 and so it was difficult to determine $\theta$. The phase boundaries are also seen in Fig. 3(b). Among the anomalies observed in Fig. 2(b), the anomalies A, B, and C are also seen. Although the anomalies $\mathrm{B}$ and $\mathrm{C}$ are faint, the anomaly $\mathrm{A}$ (the tricritical point) is clearer. It should be noted that the tilt angle monotonically increases with the increasing field inside $\mathrm{Sm}-C_{\alpha}^{*}$ in contrast to the birefringence.

In order to investigate the anomalies $\mathrm{B}$ and $\mathrm{C}$ in more detail we plotted the field dependence of $\Delta n_{\mathrm{a}}$ at each temperature. As shown in Fig. 4, at low temperatures the bire- 


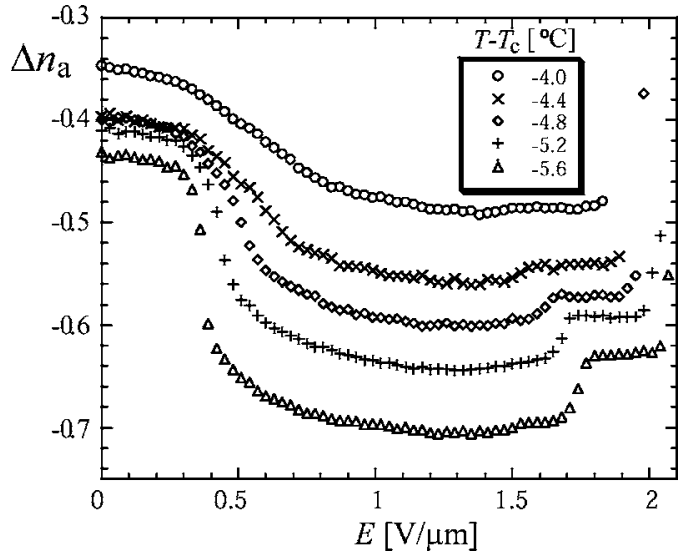

FIG. 4. Measured electric-field dependence of $\Delta n_{\mathrm{a}}$ at several temperatures.

fringence jumps at both the anomalies B and $\mathrm{C}$, and both the jumps are reduced with increasing temperature. That there exists an intermediate region with small birefringence is a most remarkable characteristic. At higher temperatures both of the changes become continuous. These discontinuous changes at low temperatures may be due to field-induced phase transitions. If the continuous changes are also due to transitions that correspond to them, there should be two tricritical points. Otherwise, there should be two critical end points. In the former case, the intermediate phase is different from the outer phases in symmetry, while in the latter case, the three phases are the same, such as the liquid-gas transition. Our theoretical consideration will support the former case. The tilt angle shows almost the same behavior with respect to the jumps, though the data are scattered.

In what follows, we clarify the molecular orientation of the intermediate phase by numerical calculations on the basis of the phenomenological discrete model. This was originally proposed to investigate the dispersion relation of orientational relaxation modes in antiferroelectric liquid crystals by Sun et al. [9]. It was later developed to study the phase transitions. We use the simplest possible free energy that describes our results,

$$
\begin{aligned}
G= & \sum_{j=1}^{N}\left[\frac{A}{2} \vec{\xi}_{j}^{2}+\frac{B}{4} \vec{\xi}_{j}^{4}+a_{1} \vec{\xi}_{j} \cdot \vec{\xi}_{j+1}+a_{2} \vec{\xi}_{j} \cdot \vec{\xi}_{j+2}+f_{1}\left(\vec{\xi}_{j} \times \vec{\xi}_{j+1}\right)_{z}\right. \\
& \left.+b\left(\vec{\xi}_{j} \cdot \vec{\xi}_{j+1}\right)^{2}-c E \xi_{j y}\right]
\end{aligned}
$$

where $\vec{\xi}_{j}$ is the projection of the director in the $j$ th layer onto the smectic layer. The first two terms are the usual meanfield expansions of the free energy for each layer. The $a_{1}$ and $a_{2}$ terms describe, respectively, the NN and NNN interlayer interactions, the $f_{1}$ term the NN chiral interaction, the $b$ term the NN nonlinear interlayer interaction. The last term describes the interaction with the electric field applied along the $x$ direction. Some physical origins of these terms have been proposed $[10,11]$.

We determined the above coefficients as follows. By rescaling $G, \vec{\xi}_{j}$, and $E$ suitably, we can put $B=a_{1}=c=1$ without

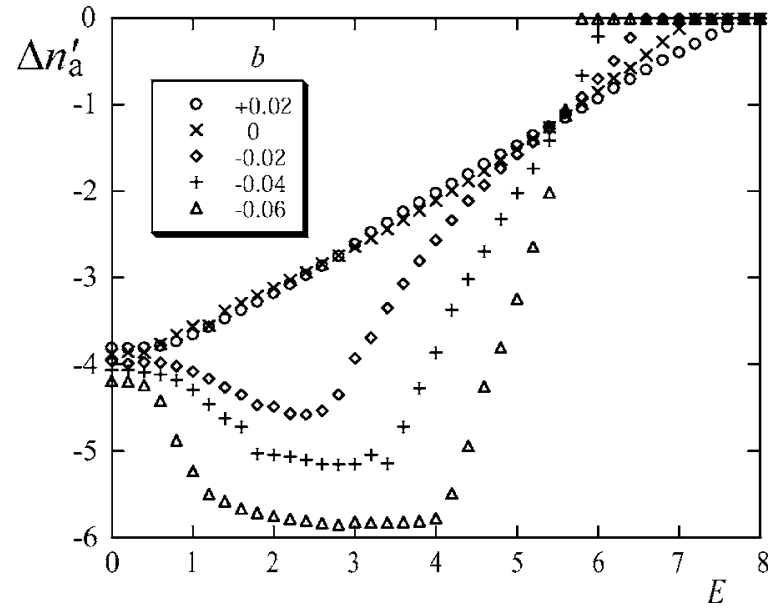

FIG. 5. Calculated electric-field dependence of $\Delta n_{\mathrm{a}}^{\prime}$ at each $b$ at $A=-1$.

loss of generality. $a_{2}$ is set to be 0.5 so that the period becomes 3 at the $\mathrm{Sm}-A-\mathrm{Sm}-C_{\alpha}^{*}$ phase-transition point under no electric field and no NN chiral interaction. We chose the small value of $f_{1}=0.01$, because $f_{1}$ may only play a role in determining the helical sense in such a phase as $\mathrm{Sm}-C_{\alpha}^{*}$ with a short pitch. For the above set of coefficients the Sm- $A-\mathrm{Sm}-C_{\alpha}^{*}$ phase transition takes place at $A \approx 1.48$ and the angle between $\vec{\xi}_{j}$ and $\vec{\xi}_{j+1}$ is about $120.2^{\circ}$ at this point. Thus, $b$ is a free coefficient that can be varied to fit the experiments, except $A$ corresponding to temperature and $E$. There may be at least two physical origins of the interaction with the coefficient $b$. One is due to quadrupolarly ordered transverse molecular dipoles [1] and the other is steric interactions [2]. The former is $b>0$, while the latter is $b<0$. To find a minimum of $G$ numerically, we used the LandauKhalatnikov equation, $\gamma \partial \vec{\xi}_{j} / \partial t=-\partial G / \partial \vec{\xi}_{j}$, where $\gamma$ is the rotational viscosity. We set $N=300$ and imposed a freeboundary condition. For a fixed set of coefficients, we made iterations starting from a random structure to reach a state of equilibrium, at which $\partial G / \partial \vec{\xi}_{j}=0$.

From the results, we calculated the birefringence $n_{\mathrm{a}}$ by using an approximate formula for a small $\left|\vec{\xi}_{j}\right|$,

$$
n_{\mathrm{a}}=\sqrt{\varepsilon}-\sqrt{\varepsilon_{\perp}}-\frac{\varepsilon_{\mathrm{a}}}{2 \sqrt{\varepsilon_{\|}}}\left[\left(1+\frac{\sqrt{\varepsilon_{\|}}}{\sqrt{\varepsilon_{\perp}}}\right)\left(\left\langle\xi_{x}^{2}\right\rangle-\left\langle\xi_{x}\right\rangle^{2}\right)+\left\langle\xi_{y}^{2}\right\rangle\right],
$$

where $\varepsilon_{\|}$and $\varepsilon_{\perp}$ are, respectively, the optical dielectric constants parallel and perpendicular to the long axis of molecules, $\varepsilon_{\mathrm{a}}=\varepsilon_{\|}-\varepsilon_{\perp}$, and $\langle\cdots\rangle$ means the spatial average, where we used the data only from $N=50$ to 250 to avoid the boundary effect. For simplicity, we used $\Delta n_{\mathrm{a}}^{\prime}$ $\equiv-\left[2\left(\left\langle\xi_{x}^{2}\right\rangle-\left\langle\xi_{x}\right\rangle^{2}\right)+\left\langle\xi_{y}^{2}\right\rangle\right]$, where we put $\varepsilon_{\|}=\varepsilon_{\perp}$ and omitted the prefactor in the above equation. Figure 5 shows the electric-field dependence of $\Delta n_{\mathrm{a}}^{\prime}$ at several values of $b$ at $A=-1$. For negative $b$, we could have an intermediate phase with small birefringence corresponding to the experimental result. The intermediate phase becomes narrower as $b$ is reduced, and around $b=0$ it disappears altogether. This indi- 


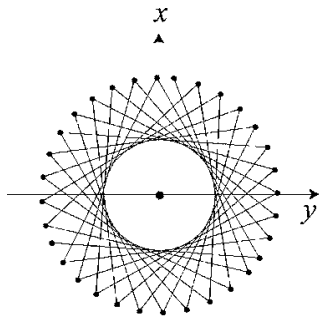

(a) $E=0$

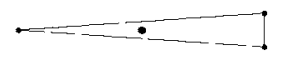

(c) $E=2.6$

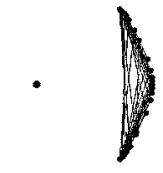

(e) $E=5.8$

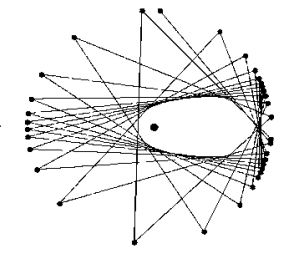

(b) $E=1.4$

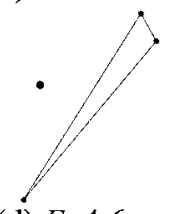

(d) $E=4.6$

(f) $E=7$
FIG. 6. Molecular orientations at several electric fields obtained on the basis of the discrete model.

cates that the intermediate phase observed in MHPOCBC may come from the $\mathrm{NN}$ nonlinear interlayer interaction with negative $b$. Although the intermediate state was reproduced in terms of the discrete model, there is a discrepancy between Figs. 4 and 5. In the experiment there is a plateau in $\Delta n_{\mathrm{a}}$ before the transition to the $\mathrm{Sm}-C$ phase (in which $\Delta n_{\mathrm{a}}$ is too large to be within the frame), while in the calculation there is a monotonic increase. This discrepancy may be ascribed to other nonlinearities.

Figure 6 shows the electric-field dependence of molecular orientation for $b=-0.04$ and $A=-1$, where dots show the tip of vector $\vec{\xi}_{j}$ and adjacent dots are linked by a line. Without field (a), there is a regular helix with a period of less than three layers. It should be noted that for $b<0$ the period decreases with decreasing temperature, i.e., $A$ in our model, while for $b>0$ it increases. Cady et al. show that the pitch decreases from $\approx 3$ to $\approx 2.6$ layers on cooling in the $\mathrm{Sm}-C_{\alpha}^{*}$ phase [5]. Therefore, this fact also supports $b<0$ for MHPOCBC. At a small field of $E=1.4$ (b), the helical structure is deformed and a modulated structure appears. At $E$ $=2.6$ (c), where the system is at the middle of the abovedescribed plateau in Fig. 5, a spatially uniform structure appears after a jump of $\Delta n_{\mathrm{a}}$. This structure lasts through a large electric-field interval. In the case of $b=-0.04$, the change from (b) to (c) is discontinuous, but we have continuous ones for $b$ near to 0 or at higher temperatures close to the $\mathrm{Sm}-A-\mathrm{Sm}-C_{\alpha}^{*}$ phase-transition point. In the plateau region, we have a spatially uniform structure with a period of just three layers. The point symmetry is $C_{2}$ with twofold axes parallel to the $y$ axis. In order to see this symmetry, one should note that $\vec{\xi}_{j}$ is the projection of the director onto the smectic layer, and so rotates the director. This structure is the same as that for $\mathrm{Sm}-C_{\mathrm{FI} 1}^{*}$ without the helix. In this state, for example, $\vec{\xi}_{j}$ is antiparallel to the $x$ axis, but $\vec{\xi}_{j+1}$ and $\vec{\xi}_{j+2}$ are nearly parallel to it. It is easily shown that for the achiral system, i.e., $Y=0$, the latter two vectors should become rigorously parallel to it and the symmetry becomes $C_{2 \mathrm{~h}}$. For $E$ $\gtrsim 3.6$ we have another three-layer structure as shown in (d), the point symmetry of which is $C_{1}$. By further increasing the field, through a modulated structure (e) we finally arrive at the ferroelectric state $(f)$. From these results, it is clear that the intermediate plateau in Fig. 5 corresponds to a stable phase symmetrically different from the outer phases.

By using a PEM we obtained a precise $E-T$ phase diagram of MHPOCBC, and found a unique field-induced phase and two tricritical points related to it. In addition, numerical calculations were made. From the results, we conclude that the intermediate region between $\mathrm{B}$ and $\mathrm{C}$ in the $E-T$ phase diagram of Fig. 2 is a three-layer phase of $C_{2}$ symmetry, whose structure is given in Fig. 6(c), and that the nonlinear NN interlayer interaction with negative $b$ is crucial to yielding this phase.

We thank Y. Suzuki of Showa Shell Sekiyu Co., Ltd for supplying the MHPOCBC.
[1] M. Škarabot, M. Čepič, B.Žekš, R. Blinc, G. Heppke, A. V. Kityk, and I. Muševič, Phys. Rev. E 58, 575 (1998).

[2] D. A. Olson, X. F. Han, A. Cady, and C. C. Huang, Phys. Rev. E 66, 021702 (2002).

[3] A. Fukuda, Y. Takanishi, T. Isozaki, K. Ishikawa, and H. Takezoe, J. Mater. Chem. 4, 997 (1994).

[4] K. Hiraoka, Y. Takanishi, H. Takezoe, A. Fukuda, T. Isozaki, Y. Suzuki, and I. Kawamura, Jpn. J. Appl. Phys., Part 1 31, 4494 (1992).

[5] A. Cady, X. F. Han, D. A. Olson, H. Orihara, and C. C. Huang, Phys. Rev. Lett. 91, 125502 (2003).
[6] V. Bourny and H. Orihara, Phys. Rev. E 63, 021703 (2001).

[7] K. Nakayama, M. Ozaki and K. Yoshino, Jpn. J. Appl. Phys., Part 1 35, 6200 (1996).

[8] V. P. Panov, N. M. Shtykov, A. Fukuda, J. K. Vij, Y. Suzuki, R. A. Lewis, M. Hird, and J. W. Goodby, Phys. Rev. E 69, 060701(R) (2004).

[9] H. Sun, H. Orihara, and Y. Ishibashi, J. Phys. Soc. Jpn. 60, 4175 (1991).

[10] M. Čepič and B. Žekš, Phys. Rev. Lett. 87, 085501 (2001).

[11] M. Čepič and B. Žekš, Mol. Cryst. Liq. Cryst. Sci. Technol., Sect. A 301, 221 (1997). 